\title{
Circulating tumor-tissue modified HPV DNA analysis for molecular disease monitoring after chemoradiation for anal squamous cell carcinoma: a case report
}

\author{
Stanley L. Liauw ${ }^{1}$, Christina H. Son ${ }^{1}$, Ardaman Shergill ${ }^{2}$, Benjamin D. Shogan ${ }^{3}$ \\ ${ }^{1}$ Department of Radiation and Cellular Oncology, University of Chicago, Chicago, IL, USA; ${ }^{2}$ Department of Medicine, University of Chicago, IL, \\ USA; ${ }^{3}$ Department of Surgery, University of Chicago, IL, USA \\ Correspondence to: Stanley L. Liauw. University of Chicago, 5758 S Maryland Ave MC9006, Chicago, IL 60637, USA. Email: sliauw@radonc.uchicago.edu.
}

\begin{abstract}
Squamous cell carcinoma (SCC) of the anus typically arises after human papillomavirus (HPV) infection. We report on the use of molecular disease monitoring using a novel blood test measuring circulating tumor-tissue -modified HPV DNA in two patients with anal cancer. Two patients with anal SCC received concurrent chemotherapy and radiation therapy (chemoRT) with curative intent, one with a T2N0 anal margin squamous cell carcinoma with a history of AIDS, and one with a T3N0 anal squamous cell carcinoma and a history of concurrent prostate cancer. HPV genotyping at diagnosis confirmed the presence of HPV16 DNA in both cases. Circulating, tumor-tissue-modified HPV DNA (TTMV-HPV DNA) was measured in the peripheral blood utilizing digital PCR at baseline and in follow-up. Disease burden was assessed post-treatment with standard anoscopy, biopsy, and PET/CT. Plasma TTMV-HPV DNA levels were elevated at diagnosis, and decreased during and after chemoRT completion in both cases. During post treatment surveillance, TTMV-HPV DNA levels correlated with disease status including one case with progressive local recurrence within 2 months, and one case with 12 months of local control both confirmed by biopsy. These case studies present the first use of circulating tumor-tissue-modified HPV DNA as a biomarker for anal cancer. Further study of this blood test an adjunct to standard treatment and monitoring is warranted in HPV-positive anal cancer.
\end{abstract}

Keywords: Anal cancer; human papillomavirus (HPV); chemoradiation; molecular disease monitoring; case report

Submitted May 26, 2021. Accepted for publication Sep 29, 2021.

doi: 10.21037/jgo-21-300

View this article at: https://dx.doi.org/10.21037/jgo-21-300

\section{Introduction}

A molecular diagnostic test with high positive and negative predictive value for cancer measurable in peripheral blood ("liquid biopsy") (1) could have significant impact on cancer diagnosis, staging, treatment, and post-treatment surveillance, and could potentially improve survival after therapy. Cancers which are etiologically related to human papillomavirus (HPV) infection present a unique opportunity for quantifying circulating amounts of HPV DNA sequences that originate in tumor tissue. Up to
$40-80 \%$ of oropharyngeal cancers (2), $60-80 \%$ of cervical cancers (3), and $80-95 \%$ of anal cancers (4), are estimated to be related to prior HPV infection. Recently, a test has been developed and validated to measure circulating tumortissue-modified HPV DNA of high-risk HPV strains in the blood (TTMV-HPV DNA), using a digital droplet PCR approach (5). In a recent prospective study of patients with p16 positive (a tissue biomarker associated with HPV infection) head and neck cancer, TTMV-HPV DNA levels were highly correlated with disease status. Here we report on the utility of this test in anal cancer, in two 
Table 1 Timelines of significant events in Case \#1 and \#2

\begin{tabular}{|c|c|c|}
\hline Case & Time & Event \\
\hline \multirow{11}{*}{$\# 1$} & 6 years before presentation & HGSIL grade $1 / 2$ of the cervix not treated \\
\hline & 1 year before presentation & HGSIL grade 3, cervical conization \\
\hline & Initial presentation & Presents with anal margin mass, growing over 8 months \\
\hline & 5 weeks & Start of CRT; P1 TTMV measurement \\
\hline & 9 weeks & T1 TTMV measurement \\
\hline & 4 months & End of (incomplete) chemoRT \\
\hline & 9 months & S2 TTMV measurement \\
\hline & 11 months & $\begin{array}{l}\text { Progressive local disease; diverting colostomy; S3 TTMV measurement; initiation of salvage } \\
\text { chemotherapy }\end{array}$ \\
\hline & 12 months & PET/CT2 and cycle 2 salvage chemotherapy \\
\hline & 14 months & S4 TTMV measurement; initiation of salvage chemoRT \\
\hline & 16 months & Interval response of disease on clinical exam during chemoRT; T2 TTMV measurement \\
\hline \multirow[t]{7}{*}{ \#2 } & 2 years before presentation & Prostate cancer diagnosis; active surveillance pursued \\
\hline & Initial presentation & Presents with rising PSA with planned surveillance prostate biopsy \\
\hline & 3 months & S1 TTMV measurement \\
\hline & 5 months & $\begin{array}{l}\text { Anoscopy with firmness and ulceration suspicious for residual cancer; PET/CT with complete } \\
\text { response; PSA 0.81; S2 TTMV measurement }\end{array}$ \\
\hline & 8 months & Exam under anesthesia \#1 with biopsy negative; S3 TTMV measurement \\
\hline & 12 months & $\begin{array}{l}\text { Exam under anesthesia \#2 with biopsy negative; PET/CT with suspicious uptake in low rectum; PSA } \\
0.37 \text {; S4 TTMV measurement }\end{array}$ \\
\hline & 15 months & Exam under anesthesia \#3 with biopsy negative; PET/CT negative; PSA 0.38; S5 TTMV measurement \\
\hline
\end{tabular}

patients with HPV-positive disease treated with concurrent chemotherapy and radiation therapy (chemoRT) (Table 1).

We present the following case in accordance with the CARE reporting checklist (available at https://dx.doi. org/10.21037/jgo-21-300).

\section{Case presentation}

\section{Case \#1}

A 48-year-old woman presented with a slow-growing, tender, anal margin mass over 8 months. Past medical 
history was significant for HIV (with history of AIDS and non-adherence with antiretroviral therapy), right eye blindness due to cytomegalovirus infection, left eye blindness due to trauma, history of CNS meningovascular tuberculosis, seizure disorder, depression, substance abuse, and smoking. Her history was also notable for HPV-16 positive cervical intraepithelial neoplasia (CIN grade 1/2) 6 years prior to presentation. Conization was suggested, but deferred until she represented 1 year prior to presentation for gynecologic examination with CIN grade 3,1 year prior to presentation with the anal mass.

On exam under anesthesia, a $2.5 \mathrm{~cm} \times 3.5 \mathrm{~cm}$ left lateral perianal margin lesion was found approximately $0.5 \mathrm{~cm}$ away from the anus with all disease external to the anal canal. Biopsy showed an invasive, moderately differentiated squamous cell carcinoma, with HPV16 DNA detected by polymerase chain reaction on microdissected formalin-fixed paraffin-embedded tumor tissue. Computed tomography (CT) scan of the chest, abdomen and pelvis showed no evidence of metastatic disease. TTMV-HPV DNA $\left(\mathrm{NavDx}_{\mathrm{x}}{ }^{\circledR}\right.$, Naveris Inc, Natick MA) testing was performed at Naveris in their CLIA-certified, CAP accredited laboratory using the Naveris platform available in the laboratory. Testing was performed on peripheral blood collected in 10-mL cell-free DNA blood collection tubes (Streck, La Vista, NE; catalog no. 218962) using a validated digital PCR (dPCR) assay. This ctHPVDNA assay distinguishes tumorderived fragmented HPV DNA from native HPV genomic DNA, regardless of tumor HPV integration status. Primers and 5 ' hydrolysis probes were designed to specifically detect amplicons within the E6 and E7 genes encoded by highrisk HPV strain 16 and E7 gene for high-risk HPV strains $18,31,33$, and 35. Measurement at baseline showed 171 TTMV-HPV-16 fragments/mL blood (Figure 1, P1).

The patient was treated with radiation therapy and concurrent chemotherapy including 5-fluorouracil (chemoRT) for T2N0 anal margin cancer. Mitomycin was not administered due to her comorbidities of AIDS (CD4 count 54, HIV viral load undetectable), frailty, malnutrition and concern for tolerance. The second planned cycle of $5 \mathrm{FU}$ was declined by the patient due to grade 4 mucositis and prolonged hospitalization needing IV pain medications after cycle 1 . Her initial radiation plan specified 41.4 Gy to pelvic and inguinal lymph nodes, lower mesorectum, anus and adjacent anal margin tumor, with sequential boost to the anus and anal margin tumor to $50.4 \mathrm{~Gy}$ at $1.8 \mathrm{~Gy} / \mathrm{fx}$ over 5.5 weeks. However, inconsistent treatment adherence resulted in delivering a total dose of only $45 \mathrm{~Gy}$ over 12 weeks. The treatment course was not prolonged by radiation toxicity, as she experienced grade 2 skin and grade 1 gastrointestinal toxicity at the conclusion of the RT course. During the course of therapy, 4 weeks after initiation (at $27 \mathrm{~Gy}$ ), there was a clinical response with a significant reduction in local pain, and only a $1.5 \mathrm{~cm}$ area of superficial ulceration with surrounding granulation tissue. At the corresponding time, TTMV-HPV16 was undetectable in the blood (Figure 1, T1). There was a residual non-healing ulcer without raised margins at weeks 8 and 12 (Figure 1, T2 and T3).

Two months after therapy, follow-up exam continued to show incompletely healed skin, with development of central firmness and a deeper, punctate area of necrosis in the setting of increasing pain; this presentation was suspicious for persistent disease. NavDx testing was positive for circulating TTMV-HPV16 DNA (21 fragments/mL, Figure 1, S1). Four months following chemoRT, she was admitted with anal pain, and an FDG positron emission tomography (PET) scan revealed increased cutaneous activity at the site of the tumor, without any distant activity (Figure 1, S2). Biopsy confirmed a local recurrence, and circulating TTMV-HPV16 was further elevated (592 fragments/mL). Seven months following chemoRT, because of severe anal pain, a plan was devised for her to undergo colonic diversion, induction chemotherapy consisting of carboplatin and paclitaxel, and then chemoRT if there was no distant progression. A NavDx test for circulating TTMV-HPV DNA levels was drawn prior to induction therapy (896 fragments/mL, Figure 1, S3) and prior to chemoRT (4,293 fragments/mL, Figure 1, S4). She is currently 16 months beyond her initial presentation, having recently completed salvage chemoRT including re-irradiation of the previously treated anal margin mass, ipsilateral pelvic and groin lymph nodes, and mesorectum. Her most recent assessment of TTMV-HPV DNA levels showed clearance of $90 \%$ (479 fragments/mL, Figure 1, T2) at day 16 of 20 of her course of salvage therapy.

\section{Case \#2}

A 73-year-old man with a history of congestive heart failure, cerebrovascular accident, morbid obesity (body mass index 45), and low-risk prostate cancer (Grade Group 1, PSA $5.5 \mathrm{ng} / \mathrm{mL}$, clinical stage $\mathrm{T} 1 \mathrm{c}, 2 / 12$ cores positive), underwent a repeat surveillance prostate biopsy via transrectal ultrasound that was interpreted as high-grade urothelial cancer with squamous differentiation in 8 of 12 

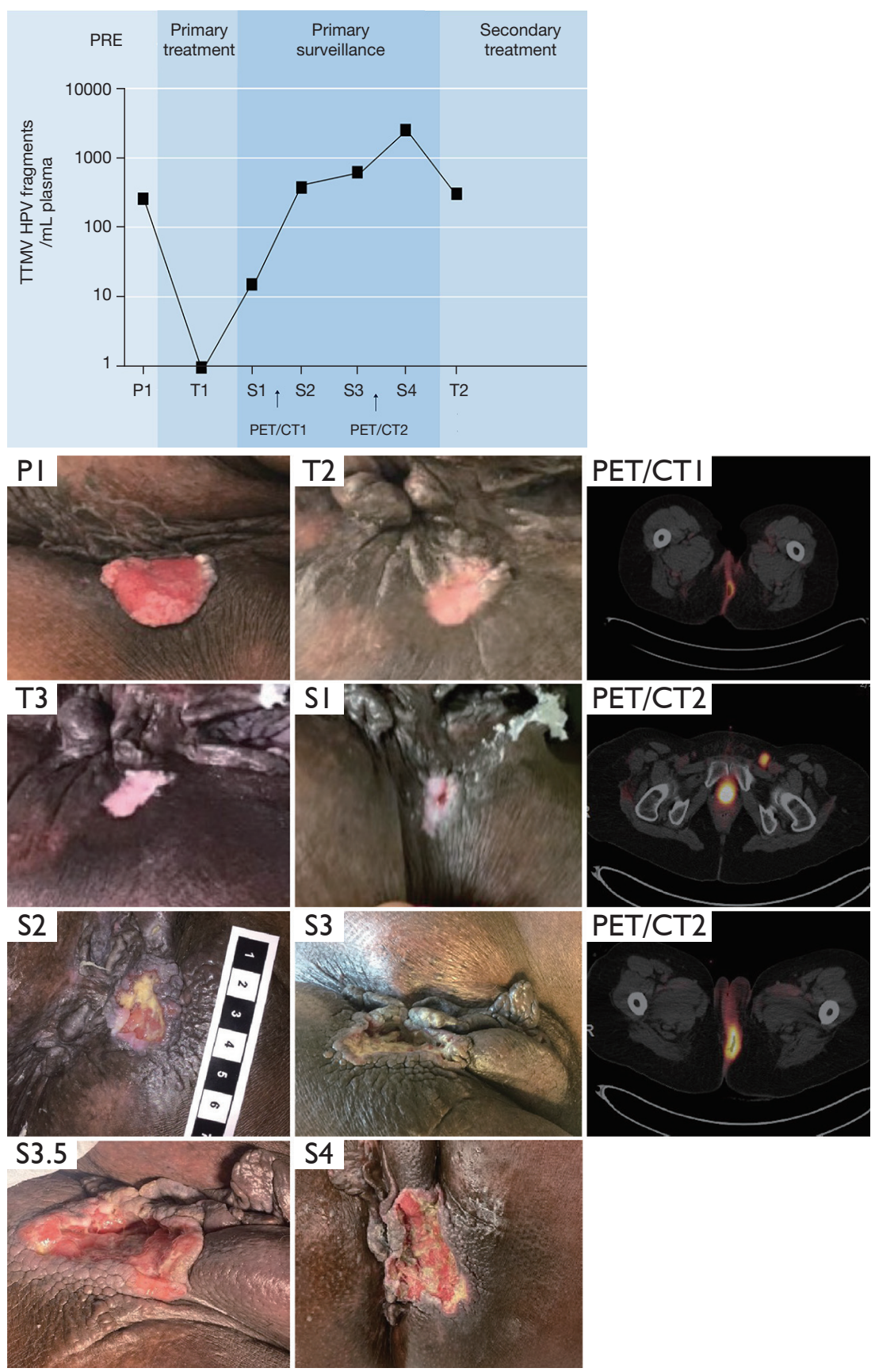

Figure 1 A 48-year-old woman with several medical co-morbidities presented with a T2N0M0 HPV-associated anal cancer. Plasma TTMV-HPV DNA values and photographs are shown at the indicated treatment time intervals: pre-treatment $(\mathrm{P})$, during treatment $(\mathrm{T})$, and post-treatment surveillance (S). At initial presentation, there was a $2.5 \mathrm{~cm} \times 3.5 \mathrm{~cm}$ left lateral anal margin mass without involvement of the anal canal. Examination improved by week 4 of treatment (T1) at which time TTMV-HPV16 DNA was undetectable. Only a superficial ulceration of the skin was observed at week 8 (T2) and week 12 of treatment (T3). Two months after therapy, she presented with pain and ulceration (S1), and TTMV-HPV16 DNA was detectable. Biopsy confirmed local recurrence. PET/CT showed hypermetabolic cutaneous activity suspicious for local tumor recurrence (PET/CT1). With further local progression at the primary site (S2, S3, S3.5) and progression on the PET/CT to involve ipsilateral pelvic and groin lymph nodes (PET/CT2), she underwent salvage chemoRT (S4), with a subsequent TTMV-HPV16 DNA drawn in her last week of therapy (T2). TTMV-HPV DNA, tumor-tissue-modified HPV DNA. 


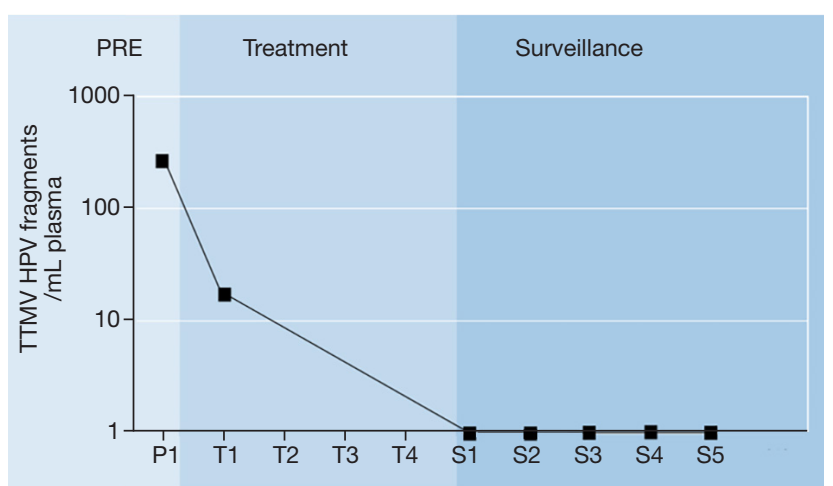

Figure 2 A 73-year-old man presented with a T1c prostate cancer and a T3N0M0 HPV-associated anal cancer. Plasma TTMVHPV DNA values are shown at the indicated treatment time intervals: pre-treatment $(\mathrm{P})$, during treatment $(\mathrm{T})$, and posttreatment surveillance $(\mathrm{S})$. At initial presentation, he had a $5.2 \mathrm{~cm}$ anorectal mass based on clinical exam, MRI, and PET/CT. Plasma TTMV-HPV16 DNA values were elevated at diagnosis $(\mathrm{P})$, decreased during treatment (T1), and undetectable at both 1 month (S1) and again 3 months after treatment completion in the setting of complete response by PET/CT (S2). Clinical exam at this time, however, was suspicious for incomplete response with a firm, ulcerated lesion on anoscopy. Exams under anesthesia at every 3 months with biopsy were negative and TTMV-HPV16 was undetectable $(\mathrm{S} 3, \mathrm{~S} 4, \mathrm{~S} 5)$. TTMV-HPV DNA, tumor-tissuemodified HPV DNA.

biopsy cores. On further workup, cystoscopy was negative for intravesical lesions, and cystoscopic biopsies showed prostatic adenocarcinoma without urothelial carcinoma. He then underwent MRI and FDG PET/CT, which demonstrated an anorectal mass measuring $5.2 \mathrm{~cm}$, and uptake in the distal rectum and anus, with equivocal activity in left obturator and right inguinal lymph nodes. Colonoscopy revealed a deeply ulcerated non-obstructing large mass in the distal rectum, extending to the anal verge and involving one-half of the lumen overlying the prostate. Biopsy of this anorectal mass demonstrated poorly differentiated squamous cell carcinoma. Immunohistochemistry revealed strong staining for p16 and $\mathrm{p} 40$, consistent with an HPV-driven squamous cell carcinoma. Because of the initial concern for urothelial cancer and question surrounding the primary origin of this cancer, next-generation sequencing was performed, which revealed mutations associated with anal cancer, including PIK3CA amplification and an E545K point mutation. Subsequently, HPV16 DNA was detected by polymerase chain reaction on microdissected formalin-fixed paraffin-embedded tumor tissue. A NavDx blood test was positive for TTMV-HPV-16 DNA (319 fragments/mL, Figure 2, P1).

He was treated with chemoRT for the T3N0 anorectal tumor, consisting of 5-FU/Mitomycin $\mathrm{C}$ at weeks 1 and 4, along with 45 Gy to pelvic and inguinal lymph nodes and mesorectum, and $54 \mathrm{~Gy}$ at $1.8 \mathrm{~Gy} / \mathrm{fx}$ to the anorectal tumor over 30 days. His prostate cancer was treated with a simultaneous integrated boost to the prostate to $66 \mathrm{~Gy}$. He completed the planned therapy without any treatment breaks over 6 weeks with grade 1 acute bladder and grade 2 acute gastrointestinal toxicity. During the course of therapy, 4 weeks after initiation (at 28.8 Gy), he had resolution of local symptoms at presentation including rectal pain and blood per rectum, and TTMV-HPV16 levels decreased to 19 fragments/mL (Figure 2, T1).

One month after completion of therapy, TTMVHPV16 DNA values were undetectable (Figure 2, S1). Three months after completion he underwent standard post-treatment evaluation including FDG PET/CT and anoscopy. PET/CT showed complete resolution of all FDG avidity, but anoscopy showed firmness and ulceration at the anus that was concerning for residual disease, while TTMV-HPV-16 was undetectable (Figure 2, S2). Because of the anoscopy finding, he was scheduled for exam under anesthesia (EUA) in another 3 months. Six months out of therapy, EUA \#1 showed a firm, ulcerated lesion in the anus suspicious for disease, but biopsy was negative for cancer. TTMV-HPV-16 was undetectable (Figure 2, S3). Nine months out of therapy, follow-up PET/CT showed new uptake in the low rectum suspicious for disease. The level of suspicion for disease recurrence was considered moderate based on the prior EUA, but on repeat EUA \#2, biopsy was again negative for cancer, showing fibrosis and reactive changes only, and TTMV-HPV-16 levels were undetectable (Figure 2, S4). Subsequent interval EUA \#3 with biopsy and PET/CT were negative 12 months after therapy. He was without any local symptoms concerning for recurrent disease and had undetectable TTMV-HPV-16 (Figure 2, $\mathrm{S} 5$ ) and biochemical control of the prostate cancer (PSA $0.38 \mathrm{ng} / \mathrm{mL})$.

All procedures performed in studies involving human participants were in accordance with the ethical standards of the institutional and/or national research committee(s) and with the Helsinki Declaration (as revised in 2013). This study was approved by the Institutional Review Board (IRB) of the University of Chicago Medical Center (IRB \#20-0410). Written informed consent was obtained 
from the patients for publication of this case report and accompanying images. A copy of the written consent is available for review by the editorial office of this journal.

\section{Discussion}

Monitoring molecular disease with HPV ctDNA testing may have value for anal and other HPV-associated cancers (6). Although promising, HPV ctDNA testing is unable to distinguish between DNA derived from cancer cells versus viral particles associated with HPV infection. This case series is the first report on the use of circulating TTMVHPV DNA longitudinally during surveillance as a biomarker in anal cancer for molecular disease monitoring using a CLIA-certified, commercially available test. TTMV-HPV DNA discriminates between the sources of HPV, thereby increasing tumor specificity by eliminating signal derived from viral particles associated with an HPV infection. In two patients who had either local recurrence or successful treatment response, TTMV-HPV DNA levels were detectable at diagnosis, and were correlated with disease burden post-treatment as determined by standard clinical evaluation (exam, anoscopy with biopsy, and PET/CT). These findings, together with the findings of others $(5,6)$, suggest that circulating tumor HPV DNA for high-risk HPV strains may play an important role in key aspects of cancer management, including screening, patient selection and risk stratification, modification of treatment based on response, and disease surveillance.

A few observations merit further discussion. First, the rise and fall of TTMV-HPV DNA levels with disease burden is encouraging to observe. Case 1 had an anal margin cancer which could be entirely visualized on external exam to monitor treatment response, with TTMVHPV DNA levels matching the patient's clinical course of local recurrence. In case 2, although post-treatment response at 6-9 months was inconclusive based on exam under anesthesia and PET/CT, the undetectable circulating TTMV-HPV DNA levels were concordant with the lack of pathologic progression through 15 months of followup to date. Provided that levels of circulating tumor HPV DNA are highly correlated with disease recurrence, molecular disease monitoring of TTMV-HPV DNA can confirm a diagnosis of recurrence in equivocal cases, and promote early detection compared to standard approaches. Surgical salvage with abdominoperineal resection is considered standard therapy for locally recurrent cases, but is associated with only $\sim 50 \% 5$-year survival (7); early salvage surgery in the setting of biopsy confirmed persistence of disease could potentially improve the chance for successful disease outcome. An important caveat to acknowledge is that a positive circulating biomarker does not discriminate between local and distant disease, but rather complements standard clinical and radiographic approaches to determine the most suitable therapeutic strategy. Second, a decline in TTMV-HPV DNA levels occurred prior to the completion of chemoRT in both cases after only 4 weeks of chemoRT, suggesting that clearance in the blood may occur prior to completion of therapy. This early response assessment could potentially inform patient candidacy regarding de-escalated treatment approaches. Such studies would be of great interest because patients treated with chemoRT consisting of 5-FU, mitomycin C, and 50.4-54 Gy for anal cancer accept significant risks of toxicity, including a $~ 40 \%$ rate of hospitalization within 3 months after chemoRT (8), as well as late side effects which can adversely affect sexual health, gastrointestinal function, and bone strength $(9,10)$. Meanwhile, local control may approach $100 \%$ in selected contemporary series treating HPV-positive disease with standard doses (11), and doses as low as 30 Gy as per the Nigro experience may be adequate for at least some patients (12). While a biomarker assessing early treatment response could provide valuable information in the selection of patients for de-escalation of therapy, it is important to note that an undetectable level of TTMV-HPV DNA after treatment does not imply clearance of disease at the primary site, and circulating tumor HPV DNA should not be used to modify treatment outside of a clinical trial.

The findings presented here in HPV-positive anal cancer appear to be consistent with the findings of a study of TTMV-HPV DNA in HPV-positive oropharyngeal squamous cell carcinoma (5). In that study, 115 patients treated with chemoRT for head and neck cancer were tested for circulating tumor HPV DNA to complement standard of care surveillance with physical exam and PET/ CT. In 28 patients (24\%) with a detectable ctHPV DNA after treatment, the 2-year relapse free survival was $30 \%$, compared to $100 \%$ for those with undetectable ctHPV DNA levels. Some patients with an abnormal posttreatment level had spontaneous clearance, but consecutive abnormal ctHPV DNA levels were highly correlated with eventual clinical failure (PPV 94\%, NPV 100\%). Median lead time from the first abnormal ctHPV DNA blood test to biopsy-proven recurrence was 3.9 months.

While additional clinical validation of this test will 
be required, and is the subject of an ongoing feasibility study in HPV-positive anal cancer and cervical cancer (NCT04857528), this case report shows that TTMVHPV DNA may serve as a useful biomarker to complement treatment and surveillance after chemoRT for anal cancer. Given that patients with HPV-positive anal cancer may benefit from efforts to de-escalate treatment intensity (13), this novel test is a promising candidate to be integrated into protocols in which treatment and surveillance methods may be adapted towards ctHPV DNA treatment response.

\section{Acknowledgments}

We gratefully acknowledge Drs. Charlotte Kuperwasser and Ian Manger (Naveris) for helpful discussions.

Funding: None.

\section{Footnote}

Reporting Checklist: The authors have completed the CARE reporting checklist. Available at https://dx.doi. org/10.21037/jgo-21-300

Conflicts of Interest: All authors have completed the ICMJE uniform disclosure form (available at https://dx.doi. org/10.21037/jgo-21-300). SLL and CHS report that Naveris has provided lab support for NCT04857528 and for article processing charges of this manuscript. The other authors have no conflicts to declare.

Ethical Statement: The authors are accountable for all aspects of the work in ensuring that questions related to the accuracy or integrity of any part of the work are appropriately investigated and resolved. All procedures performed in studies involving human participants were in accordance with the ethical standards of the institutional and/or national research committee(s) and with the Helsinki Declaration (as revised in 2013). This study was approved by the Institutional Review Board (IRB) of the University of Chicago Medical Center (IRB \#20-0410). Written informed consent was obtained from the patients for publication of this case report and accompanying images. A copy of the written consent is available for review by the editorial office of this journal.

Open Access Statement: This is an Open Access article distributed in accordance with the Creative Commons Attribution-NonCommercial-NoDerivs 4.0 International
License (CC BY-NC-ND 4.0), which permits the noncommercial replication and distribution of the article with the strict proviso that no changes or edits are made and the original work is properly cited (including links to both the formal publication through the relevant DOI and the license). See: https://creativecommons.org/licenses/by-nc-nd/4.0/.

\section{References}

1. Crowley E, Di Nicolantonio F, Loupakis F, et al. Liquid biopsy: monitoring cancer-genetics in the blood. Nat Rev Clin Oncol 2013;10:472-84.

2. Marur S, D'Souza G, Westra WH, et al. HPV-associated head and neck cancer: a virus-related cancer epidemic. Lancet Oncol 2010;11:781-9.

3. Hammer A, Rositch A, Qeadan F, et al. Age-specific prevalence of HPV16/18 genotypes in cervical cancer: A systematic review and meta-analysis. Int J Cancer 2016;138:2795-803.

4. Hoots BE, Palefsky JM, Pimenta JM, et al. Human papillomavirus type distribution in anal cancer and anal intraepithelial lesions. Int J Cancer 2009;124:2375-83.

5. Chera BS, Kumar S, Beaty BT, et al. Rapid Clearance Profile of Plasma Circulating Tumor HPV Type 16 DNA during Chemoradiotherapy Correlates with Disease Control in HPV-Associated Oropharyngeal Cancer. Clin Cancer Res 2019;25:4682-90.

6. Cabel L, Jeannot E, Bieche I, et al. Prognostic Impact of Residual HPV ctDNA Detection after Chemoradiotherapy for Anal Squamous Cell Carcinoma. Clin Cancer Res 2018;24:5767-71.

7. Mariani P, Ghanneme A, De la Rochefordière A, et al. Abdominoperineal resection for anal cancer. Dis Colon Rectum 2008;51:1495-501.

8. Pollom EL, Wang G, Harris JP, et al. The Impact of Intensity Modulated Radiation Therapy on Hospitalization Outcomes in the SEER-Medicare Population With Anal Squamous Cell Carcinoma. Int J Radiat Oncol Biol Phys 2017;98:177-85.

9. de Meric de Bellefon M, Lemanski C, Castan F, et al. Long-term follow-up experience in anal canal cancer treated with Intensity-Modulated Radiation Therapy: Clinical outcomes, patterns of relapse and predictors of failure. Radiother Oncol 2020;144:141-7.

10. Bazire L, Xu H, Foy JP, et al. Pelvic insufficiency fracture (PIF) incidence in patients treated with intensitymodulated radiation therapy (IMRT) for gynaecological or anal cancer: single-institution experience and review of the 
literature. Br J Radiol 2017;90:20160885.

11. Foster CC, Lee AY, Furtado LV, et al. Treatment outcomes and HPV characteristics for an institutional cohort of patients with anal cancer receiving concurrent chemotherapy and intensity-modulated radiation therapy. PLoS One 2018;13:e194234.

12. Nigro ND, Seydel HG, Considine B, et al. Combined

Cite this article as: Liauw SL, Son CH, Shergill A, Shogan BD. Circulating tumor-tissue modified HPV DNA analysis for molecular disease monitoring after chemoradiation for anal squamous cell carcinoma: a case report. J Gastrointest Oncol 2021;12(6):3155-3162. doi: 10.21037/jgo-21-300 preoperative radiation and chemotherapy for squamous cell carcinoma of the anal canal. Cancer 1983;51:1826-9.

13. Turchan WT, Liauw SL. Chemoradiation for Anal Cancer: Clinical Outcomes and Strategies to Optimize the Therapeutic Ratio According to HPV Status. Semin Radiat Oncol 2021;31:349-60. 